\title{
Contribución del panel de miositis en el estudio de pacientes con enfermedades pulmonares intersticiales $y$ sospecha de enfermedad del tejido conectivo
}

\author{
VERÓNICA WOLFF C.*,**, MATÍAS FLORENZANO V.*,***, FELIPE REYES C.*,***, \\ JUAN CARLOS MAYA C.**, MARÍA CAROLINA CUELLAR G.**, ALEXIS PERALTA B.****, \\ VIVIANA BALBOA C.**** y JUAN IGNACIO MÉNDEZ C.*****
}

\section{Contribution of myositis panel in the study of patients with interstitial lung disease and suspicion of connective tissue disease}

Introduction: The most common extramuscular manifestation of the idiopathic inflammatory myopathies (IIM) is interstitial lung disease (ILD) and the diagnosis is based on serum autoantibodies. The new specific and associated antibodies to IIM have helped to identify new clinical entities in the spectrum of IIM. The objective of this study is to evaluate the diagnostic contribution of a myositis antibodies panel (MP) in a cohort of Chilean patients with ILD without a definitive connective tissue disease (CTD). Materials and Methods: Starting on January 2017 we performed a MP to 111 consecutive patients with ILD and suspected CTD but without a definitive diagnosis through another diagnostic tools in the Lung-Rheumatological Program at the "Instituto Nacional del Tórax", Santiago, Chile. The clinical and serological baseline characteristics of the patients that were most frequently associated with the probability of having a positive panel were compared. Results: The MP was positive in 56 of 111 patients. Anti synthetase syndrome (ASS) was the most prevalent diagnosis. The most frequent antibodies were Ro-52, PM/Scl-75 and Ku. The most frequent variables in the positive MP group were the presence of Raynaud's phenomenon, myositis, mechanic's hands, positive Ro and La antibodies and the presence of combined pattern of nonspecific interstitial pneumonia and organizing pneumonia in chest computed tomography scan. Conclusions: The incorporation of the MP has helped us to improve our diagnostic precision of patients with CTD/ILD. We present clinical and serological elements that refine the performance of the test.

Key words: Lung Disease, Interstitial; Connective tissue Diseases; Autoantibodies; Myositis; Antisynthetase Syndrome; Rheumatic Diseases.

\section{Resumen}

Introducción: La manifestación extramuscular de las miopatías inflamatorias idiopáticas (MII) es la enfermedad pulmonar intersticial (EPI) y el diagnóstico se basa en autoanticuerpos séricos. Los nuevos anticuerpos especificos y asociados a MII han ayudado a identificar nuevas entidades clínicas en el espectro de MII. El objetivo de este estudio es evaluar la contribución diagnóstica de un panel de anticuerpos de miositis (PM) en una cohorte de pacientes chilenos con EPI sin una enfermedad del tejido conectivo (ETC) definitiva. Materiales y Métodos: A partir de enero de 2017 se realizó un panel de miositis a 111 pacientes consecutivos con EPI y sospecha de ETC, pero sin un diagnóstico definitivo

Financiamiento: Proyecto de Investigación, Servicio de Salud Metropolitano Oriente.

\footnotetext{
* Policlínico Pulmón-Reumatológico, Instituto Nacional del Tórax.

** Universidad de Chile, Departamento Medicina Oriente, Sección Reumatología.

*** Servicio Medicina Respiratoria, Instituto Nacional del Tórax.

**** Hospital del Salvador, Laboratorio de Inmunología.

${ }^{* * * * *}$ Departamento Salud Codelco Andina.
} 
a través de otra herramienta diagnóstica, en el programa de Pulmón-Reumatológico del Instituto Nacional del Tórax, Santiago, Chile. Se compararon las características basales clínicas y serológicas de los pacientes que se asociaban más frecuentemente a la probabilidad de tener un panel positivo. Resultados: El PM fue positivo en 56 de 111 pacientes. El síndrome antisintetasa (SAS) fue el diagnóstico más frecuente. Los anticuerpos más frecuentes fueron Ro-52, PM / Scl-75 y Ku. Las variables más frecuentes en el grupo PM(+) fueron la presencia del Raynaud, miositis, manos de mecánico, los anticuerpos Ro y La positivos, la presencia de un patrón combinado de neumonía intersticial inespecífica y neumonía organizada en la tomografía computarizada de tórax. Conclusiones: la incorporación del PM nos ha ayudado a mejorar nuestra precisión diagnóstica en pacientes con EPI / ETC. Presentamos elementos clínicos y serológicos que perfeccionan el rendimiento de la prueba.

Palabras clave: Enfermedad pulmonar intersticial; Enfermedades del tejido conectivo; Autoanticuerpos; Miositis; Síndrome antisintetasa; Enfermedades Reaumáticas.

\section{Introducción}

Las enfermedades pulmonares intersticiales (EPI) son un grupo heterogéneo de patologías pulmonares que comprometen principalmente el intersticio pulmonar. Se clasifican en idiopáticas cuando la causa es desconocida y en secundarias cuando están en el contexto de una enfermedad del tejido conectivo (ETC) o en relación hipersensibilidad a agentes orgánicos o inorgánicos. $\mathrm{Su}$ diagnóstico muchas veces es complejo y es necesario un equipo multidisciplinario en su abordaje ${ }^{1}$.

Las ETC son patologías autoinmunes sistémicas que entre sus manifestaciones pueden comprometer el parénquima pulmonar $\mathrm{y}$, en particular, son una causa cada vez más reconocida de $\mathrm{EPI}^{2}$. Algunos estudios muestran que son responsables de hasta el $30 \%$ de las $\mathrm{EPI}^{3}$.

Entre las ETC que con mayor frecuencia se asocian a EPI, encontramos esclerosis sistémica (ES), artritis reumatoide (AR), miopatías inflamatorias idiopáticas (MII) y los síndromes de sobreposición (SP) principalmente en el espectro de ES y MII.

En los últimos años, se ha reconocido un importante grupo de pacientes con EPI y algún elemento clínico o serológico de ETC, sin constituir una mesenquimopatía definida. Esta situación ha dado origen al concepto de neumonía intersticial con rasgos de autoinmunidad (NIRA) ${ }^{4}$.

El diagnóstico de las EPI/ETC puede ser complejo y a veces requiere de pruebas adicionales invasivas y/o poco disponibles (biopsia de piel, biopsia muscular, biopsia de glándulas salivales, biopsia pulmonar, etc). Sin embargo, este esfuerzo diagnóstico es muy importante por los efectos pronósticos y terapéuticos que conlleva. Un diagnóstico certero permite ofrecer un tratamiento inmunomodulador oportuno, destinado a controlar la enfermedad pulmonar y sistémica ${ }^{5,6}$.
Las MII constituyen un grupo de ETC con algunas características clínicas comunes que se presentan de manera variable según sea el perfil de autoanticuerpos (Ac) del paciente. Entre las manifestaciones clínicas más frecuentes encontramos inflamación muscular (miositis), artritis, fiebre, exantema característico y alta prevalencia de EPI. Esta última manifestación es especialmente frecuente en pacientes con algunos Ac relacionados a MII como son los Ac anti-sintetasa (AcAS), anti MDA-5, PM-Scl, Ku y Ro-52, y puede ser la primera o la única manifestación de una enfermedad autoinmune sistémica ${ }^{7,8}$. Los principales subgrupos clínicos de las MII incluyen: Dermatomiositis (DM), DM amiopática, DM asociada a neoplasias, Polimiositis, Miositis necrotizante y Miopatías por cuerpos de Inclusión.

Los pacientes con AcAS suelen clasificarse aparte en el grupo llamado "Síndrome antisintetasas" (SAS), ya que este grupo comparte un cuadro clínico similar caracterizado por alta prevalencia de EPI, un tipo de dermatitis particular llamada "manos de mecánico" (hiperqueratosis y resquebrajamiento de la piel en la cara lateral de los dedos de las manos), fiebre, miositis, artritis y fenómeno de Raynaud 9 .

Hasta hace poco tiempo, el perfil de Ac disponible para el diagnóstico de ETC incluía Ac antinucleares (ANA), Ac nucleares extractables (ENA), Ac anti DNA, factor reumatoideo (FR) y Ac anti péptidos cíclicos citrulinados (CCP), principalmente. Sin embargo, en los últimos años se ha hecho evidente que el espectro de ETC que presentan EPI como manifestación clínica no siempre puede ser detectado con este perfil limitado de Ac. Esto es especialmente relevante en el grupo de las MII, cuyo diagnóstico ha mejorado enormemente desde el descubrimiento de nuevos Ac que no están ampliamente disponibles para uso comercial. Estos nuevos Ac se pueden detectar por distintos métodos (Elisa, Inmunoblott, 
inmunoprecipitación, etc.) y normalmente se examinan en forma simultánea en kits comerciales que agrupan a varios de ellos ${ }^{6,7}$. Estos llamados "Páneles de miositis" (PM) incluyen Ac que se encuentran casi exclusivamente en MII (Ac específicos de MII) y otros que se encuentran en MII pero también en otras ETC (Ac relacionados a MII):

\section{Anticuerpos específicos de miosotis inflama- toria idiopática}

a. Ac antisintetasa (Ac AS): dirigidos contra las $t R N A$ sintetasas

- Anti Jo-1 (histidil-ARNt sintetasa).

- Anti PL-7 (treonil-ARNt sintetasa).

- Anti PL-12 (alanil-ARNt sintetasa).

- Anti EJ (glicil-ARNt sintetasa).

- Anti OJ (isoleucil-ARNt sintetasa).

b. Otros anticuerpos especificos de miositis inflamatoria idiopática:

- Mi-2 alfa y beta: DM clásica.

- SRP: Miositis necrotizante.

- MDA-5: DM amiopática y enfermedad pulmonar rápidamente progresiva.

- NXP2: DM asociada a neoplasias y calcinosis.

- TIF1gama: DM asociada a neoplasias.

- SAE1: DM clásica.

\section{Anticuerpos asociados a miositis:}

- Ku: SP entre ES y MII.

- PM/Scl-75: SP entre ES y MII.

- PM/Scl-100: SP entre ES y MII.

- Ro-52: asociado a DM, ES, Sjögren, LES (lupus eritematoso sistémico) y mayor riesgo de EPI.

En el año 2011 se reimpulsó en el Instituto Nacional del Tórax (INT) un equipo multidisciplinario de neumólogos junto a reumatólogos del Hospital del Salvador dedicados al estudio y tratamiento de EPI en el contexto de ETC: Policlínico de Pulmón Reumatológico. En este policlínico detectamos la necesidad de ampliar el estudio inmunológico en un grupo seleccionado de pacientes con EPI en los cuales no era posible configurar un diagnóstico reumatológico claro con las herramientas clínicas y los exámenes serológicos habitualmente disponibles. En este contexto, nuestra hipótesis fue que la realización de un PM ayudaría a mejorar la capacidad de realizar un diagnóstico reumatológico en este grupo de pacientes.

\section{Objetivos}

Nuestro objetivo principal fue determinar si la realización de un PM contribuye a configurar un diagnóstico reumatológico en un grupo de pacientes con EPI y sospecha de ETC subyacente, que no había podido ser confirmado con las herramientas de estudio disponibles en forma habitual.

El objetivo secundario fue determinar si existen características clínicas basales de los pacientes que ayuden a definir la población que tiene mayor probabilidad pre-test de tener una prueba positiva.

\section{Métodos}

Se planteó un estudio prospectivo y descriptivo. Entre los meses de enero de 2017 y marzo de 2018 se realizó un PM que determina 16 anticuerpos de miositis por técnica de Inmunoblot (Autoimmune Inflammatory Myopathies $16 \mathrm{Ag}$, Euroline $^{\circledR}$ ) en 111 pacientes seleccionados del Policlínico de Reumatología del Hospital del Salvador y/o del Policlínico de Pulmón Reumatológico del Instituto Nacional del Tórax.

Todos los pacientes tenían diagnóstico confirmado de EPI mediante tomografía computada de tórax de alta resolución (TCAR). Por los hallazgos clínicos se sospechaba una ETC subyacente (por ejemplo: artritis, fenómeno de Raynaud, manos de mecánico, patrones de EPI más frecuentes en ETC como neumonía intersticial no específica (NINE), neumonía en organización (NO) o combinación de NINE/NO y no tenían un diagnóstico reumatológico definitivo según los criterios de clasificación vigentes para las distintas ETC.

La determinación de los Ac del PM se realizó en el laboratorio de Inmunología del Hospital del Salvador y para realizar la prueba se obtuvo la firma de un consentimiento informado del paciente en todos los casos. El estudio contó con la aprobación del Comité de Ética del Servicio de Salud Metropolitano Oriente.

Es necesario destacar que el síndrome antisintetasa (SAS) no tiene criterios de clasificación validados y aceptados universalmente. En este contexto y para fines de nuestro estudio decidimos clasificar todo paciente con EPI y un AcAs como SAS, aún en ausencia de otras manifestaciones clínicas además de la EPI. Esta decisión se basó en que la EPI es una de las manifestaciones más importantes asociadas a los AcAs, por sobre otras manifestaciones clínicas extra- pulmonares.

El análisis de los resultados se utilizó estadís- 
tica descriptiva mediante frecuencias absolutas y relativas, promedios, desviación estándar y pruebas de significancia estadística (test de Fischer, $\chi^{2}$ ).

\section{Resultados}

De los 111 pacientes seleccionados en el estudio, el $72 \%$ fueron mujeres y $28 \%$ hombres, con un promedio de edad de $55 \pm 13$ años. En 49 pacientes el panel fue positivo para uno o más anticuerpos. En 19 pacientes los resultados fueron indeterminados. Analizado el contexto clínico de estos 19 pacientes, en 7 el resultado indeterminado se interpretó como positivo y en 12 como negativo. En suma, se consideró que 56 pacientes $(50,5 \%)$ tuvieron un panel de miositis "diagnóstico" (positivos + indeterminados positivos) y 55 pacientes $(49,5 \%)$ tuvieron un panel "no diagnóstico" (negativos + indeterminados negativos). En cuanto a los diagnósticos reumatológicos finales, la prueba permitió reclasificar a 15 pacientes como SAS, 5 pacientes como miopatía asociada a MDA-5, 12 en el espectro de otras MII, 11 como NIRA y 13 otras ETC (Tabla 1).

Tabla 1. Características de pacientes con panel de miositis positivo y negativo

\begin{tabular}{|c|c|c|c|c|c|}
\hline \multirow[t]{2}{*}{ Características generales } & \multicolumn{2}{|c|}{ Panel positivo $(n=56)$} & \multicolumn{2}{|c|}{ Panel negativo $(n=55)$} & \multirow{2}{*}{$\underset{\left(\chi^{2} / \text { Fisher }\right)}{\mathbf{p}}$} \\
\hline & n & $\%$ & $\mathbf{n}$ & $\%$ & \\
\hline Mujer & 40 & 71 & 40 & 73 & 0,879 \\
\hline Edad (años) & $51 \pm 13$ & & $58 \pm 13$ & & $0,013^{*}$ \\
\hline Historia de tabaquismo & 18 & 32 & 15 & 27 & 0,389 \\
\hline \multicolumn{6}{|l|}{ Características clínicas $\mathrm{n}$ (total)** } \\
\hline Fenómeno de Raynaud & 34 & 61 & $13(43)$ & 30 & $0,03 *$ \\
\hline Artritis & 19 & 34 & $10(47)$ & 21 & 0,5 \\
\hline Fiebre & 6 & 11 & $2(43)$ & 5 & 0,47 \\
\hline Miositis & 16 & 29 & $3(46)$ & 6 & $0,02 *$ \\
\hline Manos de mecánico & 22 & 39 & $4(45)$ & 9 & $<0,001^{*}$ \\
\hline Gottron & 13 & 23 & $1(46)$ & 2 & $0,01 *$ \\
\hline Rash heliotropo & 12 & 27 & $2(46)$ & 4 & $0,04 *$ \\
\hline Úlceras cutáneas & 8 & 14 & $0(49)$ & 0 & $0,02 *$ \\
\hline Esclerodactilia & 13 & 23 & $6(46)$ & 13 & 0,54 \\
\hline Telangiectasis periungueales & 26 & 46 & $10(46)$ & 22 & $0,04 *$ \\
\hline Telangiectasis palmares & 5 & 9 & $4(47)$ & 8 & 0,86 \\
\hline Manos edematosas & 4 & 7 & $3(46)$ & 6 & 0,92 \\
\hline \multicolumn{6}{|l|}{ Serología*** } \\
\hline ANA positivo $(>80)$ & 37 & 66 & $27(50)$ & 52 & 0,29 \\
\hline Anti DNA & 4 & 7 & $0(35)$ & 0 & 0,29 \\
\hline $\mathrm{RO}+$ & 32 & 57 & $2(49)$ & 4 & $<0,001^{*}$ \\
\hline $\mathrm{LA}+$ & 12 & 21 & $0(48)$ & 0 & $<0,001 *$ \\
\hline $\mathrm{SM}+$ & 4 & 7 & $3(49)$ & 6 & 1 \\
\hline $\mathrm{RNP}+$ & 8 & 14 & $3(46)$ & 7 & 0,35 \\
\hline SCL-70 + & 0 & 0 & $2(48)$ & 4 & 0,43 \\
\hline $\mathrm{JO} 1+$ & 2 & 4 & $1(50)$ & 2 & 0,51 \\
\hline Factor reumatoide + & 10 & 26 & $13(50)$ & 26 & 0,13 \\
\hline \multicolumn{6}{|l|}{ Patrones en tomografía axial computarizada } \\
\hline Neumonia intersticial usual (NIU) & 5 & 9 & 8 & 14 & 0,238 \\
\hline Neumonia intersticial no específica (NINE) & 27 & 48 & 24 & 43 & 0,384 \\
\hline Neumonia en organización (NO) & 8 & 14 & 5 & 9 & 0,36 \\
\hline $\mathrm{NINE}+\mathrm{NO}$ & 39 & 43 & 4 & 7 & $0,026^{*}$ \\
\hline Neumonia intersticial linfoide (NIL) & 1 & 2 & 0 & 0 & 0,32 \\
\hline Inclasificable & 1 & 2 & 2 & 4 & 0,163 \\
\hline Neumonitis por hipersensibilidad (NHS) & 0 & 0 & 6 & 11 & $0,008^{*}$ \\
\hline
\end{tabular}

$* \mathrm{p}<0,05$. **total: datos totales disponibles. ${ }^{* * *}$ Significado de las abreviaturas usadas en serología está señalada en el texto. 
De los pacientes con panel diagnóstico, treinta (54\%) presentaron un anticuerpo positivo, dieciocho $(32 \%)$ dos anticuerpos y ocho (15\%) tres o más anticuerpos positivos (Tabla 2). Los anticuerpos más frecuentes fueron Ro-52 (64,3\%), PMScl 75 (21,4\%), Ku (14,3\%) y PL-12 (12,5\%). La distribución general de los anticuerpos encontrados se detalla en la Figura 1.

La menor edad, la presencia de fenómeno de Raynaud, miositis, manos de mecánico, exantema de Gottron, exantema heliotropo, úlceras cutáneas, telangectasias periungueales, patrón radiológico de NINE/NO y presencia de Ro y La en la serología habitual fueron las características asociadas a una probabilidad estadísticamente significativa de presentar un panel positivo (Tabla 1).

\section{Comentarios y conclusiones}

El PM contribuyó a lograr un diagnóstico reumatológico definitivo en el 50,5\% de los casos estudiados. La certeza diagnóstica nos permitió instaurar $\mathrm{u}$ optimizar una terapia dirigida y un plan de seguimiento adecuado en estos casos.

Considerando que se trata de una prueba diagnóstica y no de seguimiento (solo se realiza una
Tabla 2. Diagnóstico final de pacientes con panel de miositis positivo

\begin{tabular}{|lcc|}
\hline Panel positivo & & \\
Diagnóstico final & n & $\%$ \\
\hline Síndrome antisintetasa & 15 & 26,8 \\
NIRA & 11 & 19,7 \\
\hline Miopatía asociada a MDA5 & 5 & 8,9 \\
\hline Escleromiositis & 5 & 8,9 \\
\hline Esclerodermia & 4 & 7,1 \\
\hline Sjögren primario & 4 & 7,1 \\
\hline Dermatomiositis amiopática & 3 & 5,4 \\
\hline Dermatomiositis & 3 & 5,4 \\
\hline LES/Esclerodermia & 3 & 5,4 \\
\hline Polimiositis & 1 & 1,8 \\
\hline LES & 1 & 1,8 \\
\hline Vasculitis ANCA MPO & 1 & 1,8 \\
\hline Total & 56 & 100 \\
\hline
\end{tabular}

NIRA: neumonía intersticial con rasgos autoinmunes, MDA5: melanoma differentiation-associated gene 5, LES: lupus eritematoso sistémico), ANCA MPO (anticuerpos anticitoplasma de neutrófilo mieloperoxidasa).

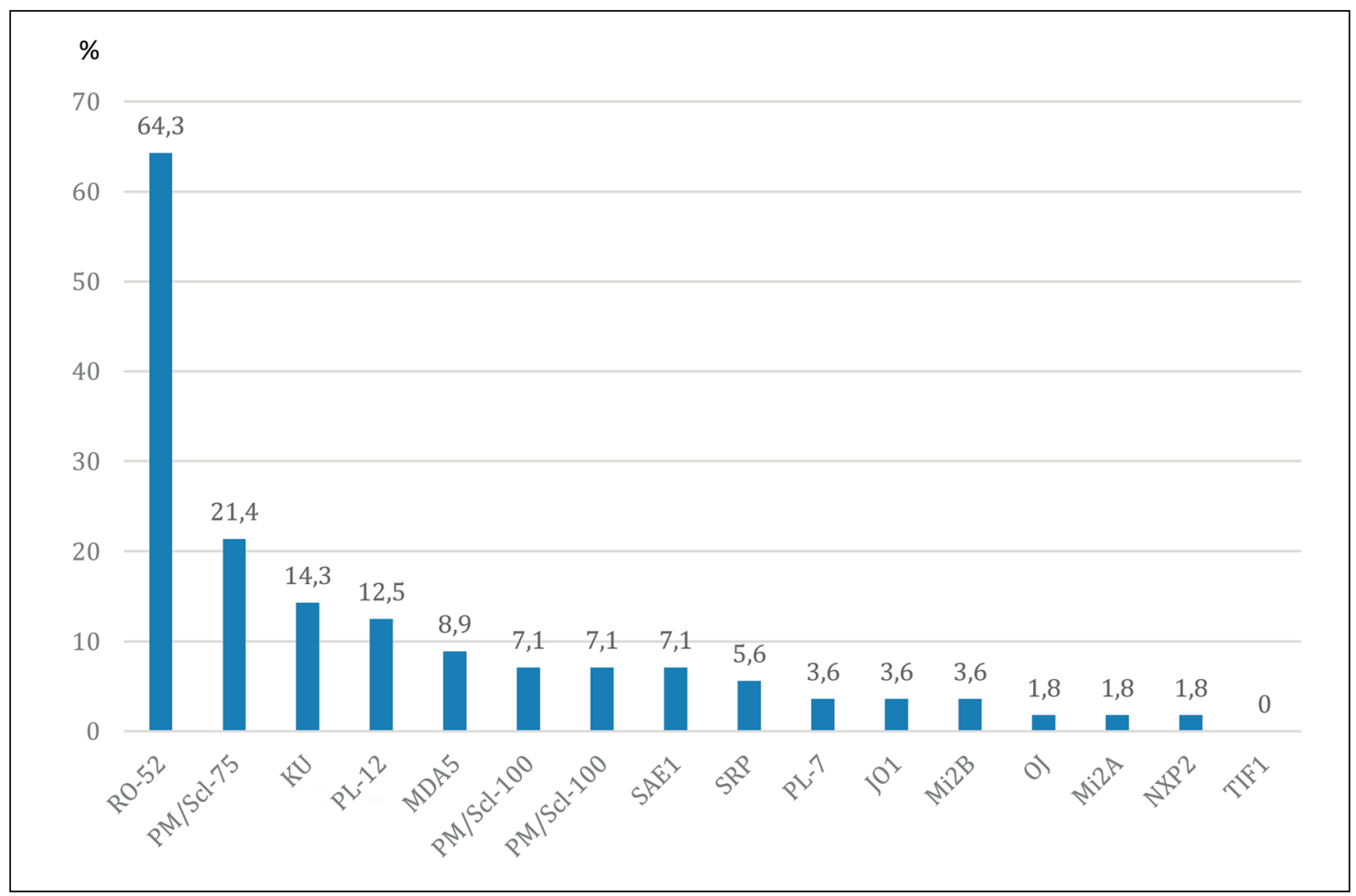

Figura 1. Distribución de anticuerpos positivos, expresados en porcentajes (\%). 
vez), creemos que se justifica realizarla en los casos en que existe una sospecha diagnóstica importante (probabilidad pretest moderada a alta), motivada principalmente por hallazgos del examen físico que orienten a una ETC en el espectro de las MII (fenómeno de Raynaud, miositis, manos de mecánico, exantema de Gottron, exantema heliotropo, úlceras cutáneas, telangectasias periungueales) o patrones radiológicos de pulmón del espectro NINE/NO y en que el estudio inicial habitualmente disponible sea no concluyente.

Es importante destacar que esta prueba no solo es útil en los casos de EPI con sospecha de ETC como etiología de base. También se ha convertido en una herramienta de mucha utilidad diagnóstica en el estudio de pacientes con ETC en el espectro de las MII sin compromiso pulmonar y con otras manifestaciones clínicas extra-musculares (artritis sero-negativas, síndrome febril prolongado, úlceras cutáneas de causa no aclarada, etc.), en los que existen muy pocas o ninguna herramienta diagnóstica serológica disponible actualmente ${ }^{6}$. En este contexto, el rendimiento del PM podría ser igual o mayor al mostrado en este estudio.

Los resultados de nuestro estudio se comparan con los publicados en otros países, difiriendo en los porcentajes de pruebas positivas según la selección de la población a estudiar. Un estudio canadiense reciente de Fidler et $\mathrm{al}^{10}$ encontró $26,7 \%$ de Ac relacionados a miositis positivos en 165 pacientes con EPI sin ETC confirmada ni sospechada. Los anticuerpos más frecuentes también fueron Ro-52, PM/Scl 75 y Jo-1. Nuestros resultados muestran pocos casos de Jo-1 porque este es un Ac que está normalmente incluido en el perfil ENA disponible habitualmente. Así, cuando Ac anti-Jol resultaba positivo, era posible realizar el diagnóstico de SAS sin necesidad realizar Ac de miositis.

En el momento de realizar este estudio, logramos que por primera vez en Chile se determinen estos Ac en un hospital del sistema público de salud. Pensamos que tener acceso a este tipo de exámenes es de vital importancia y necesidad en los hospitales de nuestro país que sean centros de referencia para este tipo de patologías.

\section{Bibliografía}

1.- TRAVIS WD, COSTABELL U, HANSELL D, KING JR TE, LYNCH DA, NICHOLSON AG, et al. An Official American Thoracic Society/European Respiratory Society Statement: Update of the International Multidisciplinary Classification of the Idiopathic Interstitial Pneumonias. Am J Respir Crit Care Med 2013; 188: 733-48.

2.- FISCHER A, LEE JS, COTTIN V. Interstitial Lung Disease Evaluation: Detecting Connective Tissue Disease. Respiration 2015; 90: 177-84.

3.- MITTOO S, GELBER AC, CRISTOPHER-STINE L, HORTON MR, LECHTZON N, DANOFF SK. Ascertainment of Collagen Vascular Disease in Patients Presenting with Interstitial Lung Disease. Respiratory Medicine 2009; 103: 1152-8.

4.- FISCHER A, ANTONIOU KM, BROWN KB, CADRANEL J, CORTE TJ, DU BOIS RM, et al. An Official European Respiratory Society/American Thoracic Society research statement: interstitial pneumonia with autoimmune features. Eur Respir J 2015; 46: 976-87

5.- FISCHER A, RICHELDI L. Cross-disciplinary Collaboration in Connective Tissue Disease-Related Lung Disease. Seminars in Respiratory and Critical Care Medicine 2014; 35: 159-65.

6.- JABLONSKI E, BHORADE S, STREK ME, DEMATTE J. Recognition and Management of Myositisassociated Rapidly Progressive Interstitial Lung Disease. Chest 2020; 158: 252-63.

7.- SATOH M, TANAKA S, CERIBELLI A, CALISE SJ, CHAN EKL. A Comprehensive Overview on Myositis-Specific Antibodies: New and Old Biomarkers in Idiopathic Inflammatory Myopathy. Clin Rev Allerg Immunol 2017; 52: 1-19.

8.- BETTERIDGE Z, MCHUGH N. Myositis-Specific Autoantibodies: an Important Tool to Support Diagnosis of Myositis. J Intern Med 2016; 280: 8-23.

9.- MAHLER M, MILLER FW, FRITZLER MJ. Idiopathic Inflammatory Myopathies and the Anti-Synthetase Syndrome: A Comprehensive Review. Autoimmunity Reviews 2014; 13: 367-71.

10.- FIDLER L, DOUBLET I, KANDEL S, FISHER JH, MITTOO S, SHAPERA S. Screening for Myositis Antibodies in Idiopathic Interstitial Lung Disease. Lung 2019; 197: 277-84.

Correspondencia a:

Dr. Matías Florenzano V.

Instituto Nacional del Tórax.

Avda. JM Infante 717, Providencia. Santiago, Chile.

Email: mflorenzano@gmail.com 